\title{
MONOGENIC INVERSE SEMIGROUPS
}

\author{
G. B. PRESTON \\ (Received 30 May 1983; revised 14 June 1985) \\ Communicated by $\Upsilon$. E. Hall
}

\begin{abstract}
We give a survey of some of the realisations that have been given of monogenic inverse semigroups and discuss their relation to one another. We then analyse the representations by bijections, combined under composition, of monogenic inverse semigroups, and classify these into isomorphism types. This provides a particularly easy way of classifying monogenic inverse semigroups into isomorphism types. Of interest is that we find two quite distinct representations by bijections of free monogenic inverse semigroups and show that all such representations must contain one of these two representations. We call a bijection of the form $a_{i} \mapsto a_{i+1}, i=1,2, \ldots, r-1$, a finite link of length $r$, and one of the form $a_{i} \mapsto a_{i+1}, i=1,2, \ldots$, a forward link. The inverse of a forward link we call a backward link. Two bijections $u: A \rightarrow B$ and $r: C \rightarrow D$ are said to be strongly disjoint if $A \cap C, A \cap D, B \cap C$ and $B \cap D$ are each empty. The two distinct representations of a free monogenic inverse semigroup, that we have just referred to, are, first, such that its generator is the union of a countable set of finite links that are pairwise strongly disjoint and whose lengths are unbounded and, second, such that its generator is the strongly disjoint union of a forward link and a backward link. One of these two kinds of representations must be a strongly disjoint part of any representation of a free monogenic inverse semigroup, the remaining part not affecting the isomorphism type. Each representation of a monogenic inverse semigroup that is not free contains a strongly disjoint part, determining it to within isomorphism, that is generated by either the strongly disjoint union of a finite link and a permutation or the strongly disjoint union of a finite link and a forward link.
\end{abstract}

1980 Mathematics subject classification (Amer. Math. Soc.): 20 M 20, 20 M 30, 20 M 50.

\section{Introduction}

A monogenic inverse semigroup is an inverse semigroup generated by a single element. If $u$ is this element, generating the inverse semigroup [ $u$, then, in $[u], u$

This paper is the text of a talk given to the Séminaire du group d'étude d'algèbre de M.-P. Malliavin, April, 1980.

(C) 1986 Australian Mathematical Society $0263-6115 / 86 \$ A 2.00+0.00$ 
has a unique inverse $v$, say, i.e. $v$ satisfies, and is the sole element in $[u]$ satisfying,

$$
u v u=u \text { and } \quad v u v=v .
$$

Moreover, $[u]=\langle u, v\rangle(=\langle\{u, v\}\rangle)$, the semigroup generated by the two elements $u$ and $v$, subject of course to the identities defining inverse semigroups.

Thus each element of $[u]$ is a product of positive powers of $u$ and its inverse $v$.

In fact every element of $[u]$ can be written in the form $v^{k} u^{l} v^{m}$, and we may also impose the conditions

$$
0 \leqslant k \leqslant l, \quad 0 \leqslant m \leqslant l, \quad l>0 ;
$$

and where, if $k$ or $m$ is zero, we interpret this to mean that the corresponding factor is not present. This result is due to L. M. Gluskin (1957).

Before giving his proof, let us make some elementary remarks. For any $x, y$ in an inverse semigroup, we have

$$
(x y)^{-1}=y^{-1} x^{-1} .
$$

Hence, for $n>0,\left(u^{n}\right)^{-1}=\left(u^{-1} \cdot u^{-1} \cdots u^{-1}\right)=\left(u^{-1}\right)^{n}$. Define, for $n>0$, therefore,

$$
u^{-n}=\left(u^{-1}\right)^{n}=\left(u^{n}\right)^{-1} .
$$

It follows that (4) also holds for negative powers. For applying (4) to $u^{-1}$, we have

$$
\left(u^{-1}\right)^{-n}=\left(\left(u^{-1}\right)^{-1}\right)^{n}=\left(\left(u^{-1}\right)^{n}\right)^{-1},
$$

i.e. $\left(u^{-1}\right)^{-n}=u^{n}=\left(u^{-n}\right)^{-1}$, using (4) again (applied to $u$ ) for the last replacement. Put $-n=m$ in this, and we have

$$
\left(u^{-1}\right)^{m}=u^{-m}=\left(u^{m}\right)^{-1},
$$

which is (4) for $m<0$.

In particular, $v^{n}$ is the unique inverse of $u^{n}$, and vice versa.

LemMA 1 (Gluskin (1957), Lemma 1.2). In the inverse semigroup [u], for any natural numbers $k, l, m$, not all zero,

$$
u^{k} v^{l} u^{m}=\left\{\begin{aligned}
u^{k+m-l}, & \text { if } k \geqslant l \leqslant m, \\
u^{k} v^{l-m}, & \text { if } k \geqslant l \geqslant m, \\
v^{l-k} u^{m}, & \text { if } k \leqslant l \leqslant m, \\
v^{l-k} u^{l} v^{l-m}, & \text { if } k \leqslant l \leqslant m .
\end{aligned}\right.
$$

Proof (Gluskin). We continue to use the convention that any power to degree zero, of $u$ or $v$, that arises, is regarded as not being present. 
Let $k \geqslant l$. Then if $l=0$, or if $m=0$, the inequalities ensure that the first two equations hold. Otherwise,

$$
\begin{aligned}
u^{k} v^{l} u^{m} & =u^{k-l+1}\left(u^{l-1} v^{l-1}\right)(v u) u^{m-1}=u^{k-l+1} v u \cdot u^{l-1} v^{l-1} \cdot u^{m-1} \\
& =u^{k} v^{l-1} u^{m-1}=\cdots .
\end{aligned}
$$

From this, by repetition if necessary, follow the first two equations of the lemma. The third follows similarly by working from the right.

For the fourth equation, suppose first that (a) $l \leqslant k+m$. Then

$$
\begin{aligned}
u^{k} v^{l} u^{m} & =u^{k} v^{k} v^{l-k} u^{l-k} u^{k+m-l}=v^{l-k} u^{l-k} u^{k} v^{k} u^{k+m-l}=v^{l-k} u^{l} v^{k} u^{k+m-l} \\
& =v^{l-k} u^{l} v^{l-m}
\end{aligned}
$$

by the second equation of the lemma, since $l \geqslant k$ and $l \geqslant m$.

Suppose secondly that (b) $l \geqslant k+m$. Set $k^{\prime}=l-k, m^{\prime}=l-m$. Then $k^{\prime} \leqslant l$ and $m^{\prime} \leqslant l$, and $l \leqslant k^{\prime}+m^{\prime}$. Hence, using the symmetry of the relationship between $u$ and $v$, we have, by (a), that

$$
\begin{aligned}
v^{l-k} u^{l} v^{l-m} & =v^{k^{\prime}} u^{l} v^{m^{\prime}}=u^{l-k^{\prime}} v^{l} u^{l-m^{\prime}} \\
& =u^{k} v^{\prime} u^{m} .
\end{aligned}
$$

Theorem 1 (Gluskin (1957), Theorem 1.3). Every element of $[u]$ can be written in the form

$$
v^{k} u^{l} v^{m}, \quad \text { with } 0 \leqslant k \leqslant l, \quad 0 \leqslant m \leqslant l, \quad l>0 .
$$

Proof (Gluskin). By Lemma 1 the result is true for all elements of the form $u^{r} v^{s} u^{t}$, with $r, s, t$ natural numbers. Applying the dual of Lemma 1 to elements of the form $v^{h} u^{i} v^{j}$, we reduce them to the form $u^{r} v^{s} u^{l}$, whence, again by Lemma 1 , the result follows. Since $[u]=\langle u, v\rangle$, it follows therefore that it remains to prove the result only for products of four powers, of $u$ and $v$ in alternation. For example, consider

$$
x=v^{k} u^{l} v^{m} u^{n}
$$

with $k, l, m, n$ all natural numbers and all non-zero. In view of the truth of the result for products with just three factors, we can assume that $k \leqslant l$ and $m \leqslant l$. Then, by Lemma 1 ,

$$
u^{l} v^{m} u^{n}= \begin{cases}u^{l+n-m}, & \text { if } m \leqslant n, \\ u^{l} v^{m-n}, & \text { if } m \geqslant n,\end{cases}
$$

which reduces $x$ to the required form. The other possibility is dealt with similarly.

\section{Free monogenic inverse semigroups}

The following model is suggested by Theorem 1 . Let $N$ denote the natural numbers, i.e. the non-negative integers. Denote by $G A$ the set

$$
G A=\{(k, l, m) \mid k, l, m \in N, k \leqslant l, m \leqslant l, l>0\},
$$


and endow $G A$ with a multiplication by defining

$$
\begin{aligned}
(k, l, m) & \left(k^{\prime}, l^{\prime}, m^{\prime}\right) \\
& =\left(k+n-l \wedge n, l+l^{\prime}+n-l \wedge n-l^{\prime} \wedge n, m^{\prime}+n-l^{\prime} \wedge n\right),
\end{aligned}
$$

where $n=m+k^{\prime}$. It may be checked that $G A$ is then an inverse semigroup and that, if we set $u=(0,1,0)$, then $G A=[u]$. We then have $v=u^{-1}=(1,1,1)$, $u^{\prime}=(0, l, 0), v^{k}=(k, k, k)$ and, if $k, l, m$ satisfy $(1.2)$,

$$
v^{k} u^{l} v^{m}=(k, l, m)
$$

Moreover,

$$
(k, l, m)^{-1}=(l-m, l, l-k) .
$$

This latter result follows by direct computation, or from Lemma 1.1, using (1). For from (1) we have

$$
\begin{aligned}
(k, l, m)^{-1} & =\left(v^{k} u^{l} v^{m}\right)^{-1}=\left(v^{m}\right)^{-1}\left(u^{l}\right)^{-1}\left(v^{k}\right)^{-1} \\
& =u^{m} v^{l} u^{k} \\
& =v^{l-m} u^{l} v^{l-k}, \quad \text { by Lemma } 1.1 \\
& =(l-m, l, l-k) .
\end{aligned}
$$

The idempotents of $G A$ are the elements,

$$
(k, k+m, m) \text {. }
$$

In particular,

$$
\begin{aligned}
& (k, l, m)(k, l, m)^{-1}=(k, l, l-k), \\
& (k, l, m)^{-1}(k, l, m)=(l-m, l, m) .
\end{aligned}
$$

Gluskin's paper (1957) was devoted to classifying the different possible kinds of monogenic inverse semigroup, and he did this by investigating what equations could hold connecting two of the elements $v^{k} u^{l} v^{m}$. The existence of the model $G A$ shows that all the elements $v^{k} u^{l} v^{m}$, satisfying (1.2), may be distinct. It follows that, since there is a free inverse semigroup on one generator, $G A$ is this free inverse semigroup.

In a later paper (1961) Gluskin approached the same topic afresh and began with the consideration, not of an arbitrary monogenic inverse semigroup $[u]$, but with the semigroup that we now define and call $G B$. Let $Z$ denote the set of integers, and, as before, $N$ the non-negative integers. Then define

$$
G B=\{(k, l, m) \in N \times Z \times N \mid k+l \in N, l+m \in N, k+l+m>0\}
$$

with the multiplication

$$
(k, l, m)\left(k^{\prime}, l^{\prime}, m^{\prime}\right)=\left(k \vee\left(k^{\prime}-l\right), l+l^{\prime}, m^{\prime} \vee\left(m-l^{\prime}\right)\right) .
$$


It may be straightforwardly checked that $G B$ is an inverse semigroup generated by the element $u=(0,1,0)$. Here $v=u^{-1}=(1,-1,1), u^{l}=(0, l, 0), v^{l}=(l,-l, l)$ and, if $k, l, m$ satisfy (1.2),

$$
v^{k} u^{l} v^{m}=(k, l-m-k, m)
$$

Moreover,

$$
(k, l, m)^{-1}=(k+l,-l, l+m),
$$

and the idempotents of $G B$ are precisely the elements

$$
(k, 0, m) \text {. }
$$

In particular,

$$
\begin{aligned}
& (k, l, m)(k, l, m)^{-1}=(k, 0, l+m) \\
& (k, l, m)^{-1}(k, l, m)=(k+l, 0, m) .
\end{aligned}
$$

The product in the semigroup $G B$ is simpler than that in the semigroup $G A$, but the representation of $v^{k} u^{l} v^{m}$ by $(k, l-m-k, m)$ in $G B$ as against $(k, l, m)$ in $G A$ is a little more artificial. As has been implied by the discussion we have

LEMMA 1. $G A$ is isomoprhic to $G B$ under the isomorphism

$$
(k, l, m) \mapsto((k, l-m-k, m)),
$$

the inverse of which is

$$
((k, l, m)) \mapsto(k, k+l+m, m) .
$$

(We have used double parentheses to indicate elements of $G B$, retaining single parentheses for elements of $G A$.)

In his papers (1957) and (1961) Gluskin was discussing, except for a generalization that we shall refer to shortly, solely monogenic inverse semigroups. In (1973) H. E. Scheiblich gave a construction for an arbitrary free inverse semigroup with, of course, as a special case that of the free monogenic inverse semigroup. For the monogenic inverse semigroup Scheiblich's construction can be regarded as interpreting the element $v^{k} u^{l} v^{m}$ of $[u]$ as an ordered pair consisting of a finite interval of integers, straddling the origin, together with some integer from this interval. Indeed, set

$$
S C=\{([-a, b] ; n) \mid a, b \in N, a+b>0, n \in[-a, b]\},
$$

where $[x, y]$ is defined, for $x, y \in N$, to be the set of integers $\{z \mid x \leqslant z \leqslant y\}$, and endow $S C$ with a product thus:

$$
([-a, b] ; n)\left(\left[-a^{\prime}, b^{\prime}\right] ; n^{\prime}\right)=\left([-a, b] \cup\left(n+\left[-a^{\prime}, b^{\prime}\right]\right) ; n+n^{\prime}\right) .
$$

Then $S C$ becomes thereby an inverse semigroup generated by $u=([0,1] ; 1)$. Then $v=u^{-1}=([-1,0] ;-1), u^{l}=([0,1] ; l), v^{l}=([-l, 0] ;-l)$, and, if $k, l, m$ satisfy

$$
v^{k} u^{l} v^{m}=([-k, l-k] ; l-k-m)
$$


Moreover,

$$
([-a, b] ; n)^{-1}=([-n-a,-n+b] ; n)=(-n+[-a, b] ;-n) .
$$

The idempotents of $S C$ are just the elements

$$
([-a, b] ; 0) \text {. }
$$

In particular,

$$
\begin{aligned}
& ([-a, b] ; n)([-a, b] ; n)^{-1}=([-a, b] ; 0) \\
& ([-a, b] ; n)^{-1}([-a, b] ; n)=(-n+[-a, b] ; 0)
\end{aligned}
$$

LEMMA 2. The mappings

$$
(k, l, m) \mapsto([-k, l-k] ; l-k-m)
$$

and

$$
((k, l, m)) \mapsto([-k, l+m] ; l)
$$

are isomorphisms of GA upon $S C$ and of $G B$ upon $S C$, respectively. The inverses of $(10 \mathrm{a})$ and $(10 \mathrm{~b})$ are $\left(10 \mathrm{a}^{\prime}\right)$ and $\left(10 \mathrm{~b}^{\prime}\right)$, respectively, given by

$$
([-a, b] ; n) \mapsto(a, a+b, b-n)
$$

and

$$
([-a, b] ; n) \mapsto((a, n, b-n))
$$

We commented earlier in this section that $G A$ must be the free inverse semigroup on one generator. That it is free also follows from its isomorphism with $S C$, once it has been shown that $S C$ is free. We now give another way of showing freedom directly, and the following lemma is what we need.

LeMMA 3. Let $[u]$ be any monogenic inverse semigroup. Let $w$ and $w^{\prime}$ be any two elements of $[u]$, so that, by Theorem 1.1, there exist $k, l, m$ and $k^{\prime}, l^{\prime}, m^{\prime}$, each satifying conditions (1.2), such that $w=v^{k} u^{l} v^{m}$, and $w^{\prime}=v^{k^{\prime}} u^{\prime} v^{m^{\prime}}$. Then $w w^{\prime}=w^{\prime \prime}$ $=v^{k^{\prime \prime}} u^{l^{\prime \prime}} v^{m^{\prime \prime}}$, where

$$
\begin{aligned}
k^{\prime \prime} & =k+n-l \wedge n, \\
l^{\prime \prime} & =l+l^{\prime}+n-l \wedge n-l^{\prime} \wedge n, \\
m^{\prime \prime} & =m^{\prime}+n-l^{\prime} \wedge n,
\end{aligned}
$$

and where $n$ is written for $m+k^{\prime}$. 
Proof (after Eberhart and Selden (1972)). Set $x=v^{k}, y=u^{l}, z=v^{m}, x^{\prime}=v^{k^{\prime}}$, $y^{\prime}=u^{l^{\prime}}, z^{\prime}=v^{m^{\prime}}$. Then, applying Lemma 1.1, we obtain

$$
\begin{aligned}
y\left(z x^{\prime}\right) y^{\prime} & =u^{\prime} v^{m+k^{\prime}} u^{l^{\prime}}=u^{\prime} v^{n} u^{l^{\prime}} \\
& = \begin{cases}u^{l+l^{\prime}-n}, & \text { if } l \geqslant n \leqslant l^{\prime}, \\
u^{\prime} v^{n-l^{\prime},} & \text { if } l \geqslant n \geqslant l^{\prime}, \\
v^{n-1} u^{\prime}, & \text { if } l \leqslant n \leqslant l^{\prime}, \\
v^{n-l} u^{n} v^{n-l}, & \text { if } l \leqslant n \geqslant l^{\prime} .\end{cases}
\end{aligned}
$$

Thus

$$
\begin{aligned}
w w^{\prime} & =x\left(u^{l} v^{n} u^{l^{\prime}}\right) z^{\prime} \\
& =v^{k}\left(u^{l} v^{n} u^{l^{\prime}}\right) v^{m^{\prime}} \\
& = \begin{cases}v^{k} u^{l+l^{\prime}-n} v^{m^{\prime}}, & \text { if } l \geqslant n \geqslant l^{\prime}, \\
v^{k} u^{\prime} v^{m^{\prime}+n-l^{\prime}}, & \text { if } l \geqslant n \geqslant l^{\prime}, \\
v^{k+n-l} u^{\prime} v^{m^{\prime}}, & \text { if } l \leqslant n \leqslant l^{\prime}, \\
v^{k+n-l} u^{n} v^{m^{\prime}+n-l^{\prime}}, & \text { if } l \leqslant n \geqslant l,\end{cases}
\end{aligned}
$$

It is now a straightforward matter to check that the given formula covers all these cases.

As a corollary we have

THEOREM 1. Each of the semigroups $G A, G B$ and $S C$ is a free inverse semigroup on one generator.

Proof. Let us work with $G A$. Consider the mapping

$$
(k, l, m) \mapsto v^{k} u^{l} v^{m}
$$

of $G A$ into [ $u$ ], an arbitrary monogenic inverse semigroup. Because of Lemma 4, and because of the definition of multiplication in $G A$, this mapping is the unique extension to $G A$ of the mapping $(0,1,0) \mapsto u$, and by Lemma 4 it is a morphism. Hence $G A$ is free.

D. B. McAlister and R. McFadden also construct free, and hence free monogenic, inverse semigroups in their paper (1974), as a special case of what are now called their $P$-semigroups (1974). W. D. Munn (1974), independently of Scheiblich, found a graph-theoretic representation of free inverse semigroups which we now describe for the special case of monogenic semigroups.

In general Munn used what he called birooted trees, but for the one-generator case, these reduce to a straight line, divided into a finite number of segments, separated by vertices, their end-points, and with two distinguished vertices, 
Consider an element $v^{k} u^{l} v^{m}$ of $G A$. Munn's construction associates with this an initial vertex $\alpha$, say, and a final vertex $\beta$, say. Starting from $\alpha$ (let us consider the line of segments as being horizontally drawn on the page), the (line) graph is constructed by first drawing $k$ equal segments to the left, and then moving to the right through $l$ segments. Remembering that $l \geqslant k$, this takes us either back to the starting vertex $\alpha$, or gives us $l-k$ segments drawn to the right of $\alpha$. Finally, to obtain the vertex $\beta$, we move again to the left through $m$ segments.

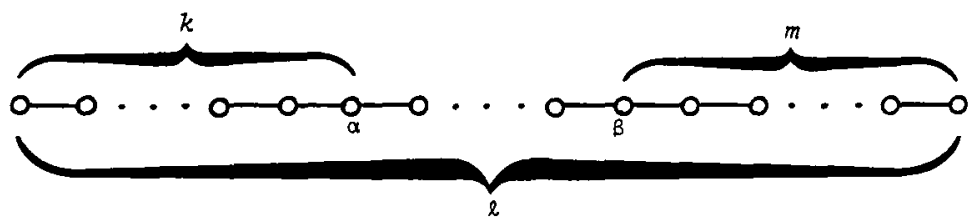

The number $k$, the first component of $(k, l, m)$, represents the number of segments to the left of the initial vertex $\alpha, l$ represents the length of (total number of segments in) the line, and $m$ represents the number of segments to the right of the final vertex $\beta$.

The product of two elements $(k, l, m)$ and $\left(k^{\prime}, l^{\prime}, m^{\prime}\right)$ of $G A$ can be obtained from their graphs: just make the initial vertex $\alpha^{\prime}$ of the graph of $\left(k^{\prime}, l^{\prime}, m^{\prime}\right)$ coincide with the final vertex $\beta$ for $(k, l, m)$, and superimpose the graph of $\left(k^{\prime}, l^{\prime}, m^{\prime}\right)$ upon that of $(k, l, m)$, regarding $\alpha$ as the initial and $\beta^{\prime}$ as the final vertex of the graph of the product $(k, l, m)\left(k^{\prime}, l^{\prime}, m^{\prime}\right)$ in $G A$. Then the first component of the product is the number of segments to the left of $\alpha$ in the superposed pair of graphs, the second component is the total length of the graphs, and the third component is the number of segments to the right of $\beta^{\prime}$.

Apart from giving this mechanical way of computing products, Munn's representation has a further advantage. In discussing $G A, G B$ and $S C$ we worked in terms of canonical forms, unique ways of expressing each element of the semigroup. But in practice the elements of a free inverse semigroup do not turn up in their canonical forms and have to be reduced to them. Munn's construction provides an easy way to do this. For Munn proved, and the generalization of this is the key to the usefulness of his construction for the general free inverse semigroup, that you always get the same graph for any two representations of the same word. For example $v^{3} u^{5} v^{5}=\left(v^{3} u^{3}\right)\left(u^{2} v^{2}\right) v^{2}=\left(u^{2} v^{2}\right)\left(v^{3} u^{3}\right) v^{2}=u^{2} v^{5} u^{3} v^{2}$, since idempotents commute. The graph for $u^{2} v^{5} u^{3} v^{2}$ is formed, starting with $\alpha$, by first drawing two segments to the right, for $u^{2}$, then going five segments to the left, so going back over the first two, and then drawing another three, for $v^{5}$; then back to the right across three segments, for $u^{3}$, and finally going two left to reach the terminal vertex $\beta$, for the final factor $v$. 


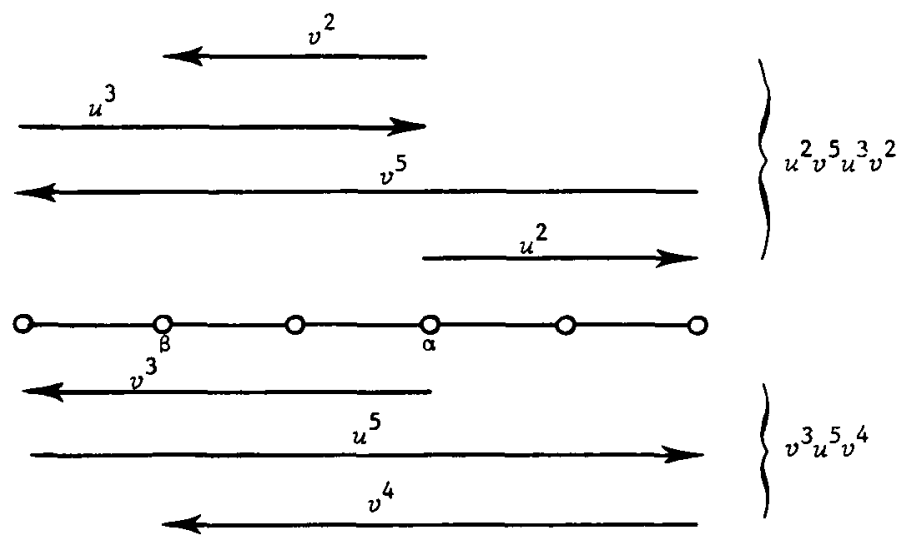

We get the same graph for $v^{3} u^{5} v^{4}$ formed in the canonical way that we began by describing.

Another approach, due to the author, to free inverse semigroups, may be found in Preston (1973). When applied to monogenic inverse semigroups this approach equivalently identifies the free monogenic inverse semigroup as the set

$$
F=\{(-l, n, m) \mid l, n \in N, m \in Z, n+l>0,-l \leqslant m \leqslant n\}
$$

endowed with a multiplication

$$
(-l, n, m)\left(-l^{\prime}, n^{\prime}, m^{\prime}\right)=\left(-l \wedge\left(m-l^{\prime}\right), n \wedge\left(m+n^{\prime}\right), m+m^{\prime}\right) .
$$

This description is virtually identical with that of Scheiblich (see (9)) described earlier.

\section{Possible generalizations}

In the semigroups $G A$ and $G B$ the operations + and - , and $\vee$ for maximum, on the integers, are used to define the product. In his paper (1961) Gluskin begins by considering an arbitrary totally ordered abelian group $G$, say, and defines a product on $G^{3}=G \times G \times G$ by

$$
(k, l, m)\left(k^{\prime}, l^{\prime}, m^{\prime}\right)=\left(k \vee\left(k^{\prime}-l\right), l+l^{\prime}, m^{\prime} \vee\left(m-l^{\prime}\right)\right),
$$

a form identical to that used to define $G B$ earlier. He notes that $G^{3}$ thereby becomes an inverse semigroup, in which, as for $G B$,

$$
(k, l, m)^{-1}=(k+l,-l, l+m),
$$


and idempotents are the elements

$$
(k, 0, m) \text {. }
$$

Moreover $G^{3}$ has an inverse subsemigroup $H^{*}(G)$, defined by $H^{*}(G)=\left\{(k, l, m) \in G^{3} \mid k \geqslant 0, m \geqslant 0, k+l \geqslant 0, l+m \geqslant 0, k+l+m>0\right\}$. When $G=Z$, then $H^{*}(G)=G B$.

Let us look at associativity more closely. The definition of product gives

$$
\begin{aligned}
((k, l, m) & \left.\left(k^{\prime}, l^{\prime}, m^{\prime}\right)\right)\left(k^{\prime \prime}, l^{\prime \prime}, m^{\prime \prime}\right) \\
=\left(\left(k \vee\left(k^{\prime}-l\right)\right) \vee\left(k^{\prime \prime}-\left(l+l^{\prime}\right)\right),\left(l+l^{\prime}\right)\right. & +l^{\prime \prime}, \\
m^{\prime \prime} & \left.\vee\left(\left(m^{\prime} \vee\left(m-l^{\prime}\right)\right)-l^{\prime \prime}\right)\right)
\end{aligned}
$$

and

$$
\begin{aligned}
(k, l, m) & \left(\left(k^{\prime}, l^{\prime}, m^{\prime}\right)\left(k^{\prime \prime}, l^{\prime \prime}, m^{\prime \prime}\right)\right) \\
=\left(k \vee\left(\left(k^{\prime} \vee\left(k^{\prime \prime}-l^{\prime}\right)\right)-l\right), l\right. & +\left(l^{\prime}+l^{\prime \prime}\right), \\
& \left.\left(m^{\prime \prime} \vee\left(m^{\prime}-l^{\prime \prime}\right)\right) \vee\left(m-\left(l^{\prime}+l^{\prime \prime}\right)\right)\right) .
\end{aligned}
$$

If $k, l, m$ belong to an additive group, then for these to be equal we require

$$
\begin{gathered}
\left(k \vee\left(k^{\prime}-l\right)\right) \vee\left(k^{\prime \prime}-\left(l+l^{\prime}\right)\right)=k \vee\left(\left(k^{\prime} \vee\left(k^{\prime \prime}-l^{\prime}\right)\right)-l\right), \\
m^{\prime \prime} \vee\left(\left(m^{\prime} \vee\left(m-l^{\prime}\right)\right)-l^{\prime \prime}\right)=\left(m^{\prime \prime} \vee\left(m^{\prime}-l^{\prime \prime}\right)\right) \vee\left(m-\left(l^{\prime}+l^{\prime \prime}\right)\right)
\end{gathered}
$$

As identities, required to hold for associativity, these two are the same, provided that addition is commutative. They both hold when, for all $k^{\prime}, k^{\prime \prime}, l^{\prime}, l$, we have

$$
\left(k^{\prime} \vee\left(k^{\prime \prime}-l^{\prime}\right)\right)-l=\left(k^{\prime}-l\right) \vee\left(k^{\prime \prime}-\left(l+l^{\prime}\right)\right) \text {; }
$$

in other words, it suffices that the partial order, with respect to which finite least upper bounds exist, is compatible with subtraction.

Any partially ordered group, i.e. a group with a compatible partial order, and which forms a semilattice (upper or lower) under this partial order, is necessarily a lattice, indeed a distributive lattice, and addition and subtraction are compatible with the meet and join operations of the lattice. This holds whether or not the group operation is commutative. (See Dubriel-Jacotin, Lesieur and Croisot (1953), Theorems 12 and 17, pp. 147-148.) Hence, for $G$ any partially ordered abelian group such that the partial order forms a semilattice (and hence a lattice), the operation we have defined on $G^{3}$ makes $G^{3}$ an inverse semigroup of which $H^{*}(G)$ is an inverse subsemigroup.

Similar remarks apply to generalizing $G A$. However, $G A$ can be generalized in a quite different way, the way that Eberhart and Selden (1972) approached what they called one-parameter inverse semigroups, of which monogenic inverse semigroups with identity are a special case. 
Eberhart and Selden begin with the multiplicative group $R^{+}$of positive real numbers, they take a subgroup $G$ of $R^{+}$and let $P=\{x \in G \mid x \geqslant 1\}$. For each $P$ they construct the free inverse semigroup $F_{P}$ generated by $P$, in the sense that there is a morphism $f: P \rightarrow F_{P}$ such that $P f$ generates $F_{P}$, and that, if $g: P \rightarrow S$ is a morphism from $P$ to any inverse semigroup $S$, then there exists a unique morphism $\theta$, say, making the following diagram commute.

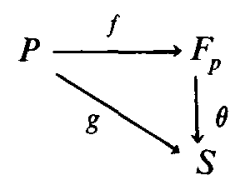

In fact $F_{P}$ has a formal structure directly analogous to $G A$ and is indeed one of the generalizations of $G A$ for which the group $G$ is totally ordered, as indicated earlier.

There is an interesting comment on the connexion between the Eberhart-Selden approach to monogenic inverse semigroups and the Scheiblich construction $C S$ in the review by D. B. McAlister (1973).

\section{Representations}

Our main purpose is to analyse the representations of monogenic inverse semigroups. We then use our results to classify the isomorphism types of monogenic inverse semigroups, providing thereby an alternative approach to the results of Gluskin (1957), (1961), and also showing which of the types he listed actually occur.

We first establish some facts about the representations of monogenic inverse semigroups that we shall find convenient to use later. In his paper (1957) Munn considered the representations, by bijective mappings, of elements of a finite inverse semigroup. The situation for elements of an infinite inverse semigroup can be dealt with very similarly.

Following Munn, let us call a finite link of length $s$ a mapping

$$
\lambda_{s}=\left(\begin{array}{llll}
a_{1} & a_{2} & \cdots & a_{s-1} \\
a_{2} & a_{3} & \cdots & a_{s}
\end{array}\right),
$$

where $\left\{a_{1}, a_{2}, \ldots, a_{s}\right\}$ is a set with $s$ elements, and where the notation means that $\lambda_{s}: a_{i} \mapsto a_{i+1}$, for $i=1,2, \ldots, s-1$. Then, for $1 \leqslant t<s$, we have

$$
\lambda_{s}^{t}=\left(\begin{array}{cccc}
a_{1} & a_{2} & \cdots & a_{s-t} \\
a_{1+t} & a_{2+t} & \cdots & a_{s}
\end{array}\right)
$$

and $\lambda_{s}^{t}=\square$, the empty mapping, for $t \geqslant s$. 
The inverse of $\lambda_{s}$ is also a finite link of length $s$. To see this, merely observe that

$$
\lambda_{s}^{-1}=\left(\begin{array}{cccc}
a_{s} & a_{s-1} & \cdots & a_{2} \\
a_{s-1} & a_{s-2} & \cdots & a_{1}
\end{array}\right)
$$

A mapping $\lambda$ of the form

$$
\lambda=\left(\begin{array}{ccccc}
a_{1} & a_{2} & \cdots & a_{k} & \cdots \\
a_{2} & a_{3} & \cdots & a_{k+1} & \cdots
\end{array}\right),
$$

where $a_{1}, a_{2}, \ldots, a_{k}, \ldots$, us a countably infinite sequence of distinct elements, will be called a forward link. Here

$$
\begin{aligned}
\lambda^{t} & =\left(\begin{array}{ccc}
a_{1} & a_{2} & \ldots \\
a_{1+t} & a_{2+t} & \ldots
\end{array}\right) \\
& =\left(\begin{array}{ccc}
a_{1} & a_{1+t} & \ldots \\
a_{1+t} & a_{1+2 t} & \ldots
\end{array}\right) \cup\left(\begin{array}{ccc}
a_{2} & a_{2+t} & \ldots \\
a_{2+t} & a_{2+2 t} & \ldots
\end{array}\right) \cup \cdots \cup\left(\begin{array}{ccc}
a_{t} & a_{2 t} & \ldots \\
a_{2 t} & a_{3 t} & \ldots
\end{array}\right),
\end{aligned}
$$

a union of $t$ forward links. Notice that any two of these forward links have disjoint domains and disjoint ranges and, moreover, are such that each domain is disjoint from each range of the other links. Any two bijections $u: A \rightarrow B$ and $v$ : $C \rightarrow D$ with these properties, i.e. such that $A \cap C, B \cap D, A \cap D$ and $B \cap C$ are each empty, or, equivalently, such that $(A \cup B) \cap(C \cup D)$ is empty, will be said to be strongly disjoint.

Notice that $\lambda^{-1}$ is not a forward link. A property of a forward link is that the domain of all powers $\lambda^{t}, t \geqslant 1$, is the same as that of $\lambda$, while the range of $\lambda^{t}$ has $t$ less elements than that of $\lambda$. A mapping of the form of $\lambda^{-1}$, viz.

$$
\mu=\left(\begin{array}{cccc}
b_{2} b_{3} b_{4} & \cdots & b_{k+1} & \cdots \\
b_{1} b_{2} b_{3} & \cdots & b_{k} & \cdots
\end{array}\right)
$$

will be called a backward link. The range of all powers of $\mu$ is the same as that of $\mu$. As for forward links, the power $\mu^{t}$ of the backward link $\mu$, is the union of $t$ strongly disjoint backward links.

Forward and backward links will be called infinite links; infinite links and finite links will generally be called links.

Lemma 1. Let $u: A \rightarrow B$ be a bijection of $A$ upon $B$. Then $u$ can be decomposed into a strongly disjoint union of a uniquely determined set of finite links, forward links, backward links, and a permutation.

Proof. Set $X=A \backslash B, Z=B \backslash A$ and $Y=A \cap B$.

Let $a_{1} \in X$. If $a_{1} u \in Z$, then $\left(a_{1} u\right) u$ does not exist. If $a_{1} u \in Y$, then $a_{1} u^{2}$ exists. Write $a_{1} u^{i}=a_{1+i}$. There are two possibilities: either (a) there exists a least $r(>1)$ such that $a_{1} u^{r}$ does not exist, or (b) all $a_{1} u^{s}$ exist. In case (a), part of the 
mapping $u$ is the finite link of length $r$

$$
\lambda=\left(\begin{array}{lll}
a_{1} & \cdots & a_{r-1} \\
a_{2} & \cdots & a_{r}
\end{array}\right) .
$$

Here, $a_{2}, a_{3}, \ldots, a_{r-1}$ all belong to $Y$, and $a_{r} \in Z$. In case (b), we get an (infinite) forward link

$$
\lambda=\left(\begin{array}{llll}
a_{1} a_{2} & \cdots & a_{k} & \cdots \\
a_{2} a_{3} & \cdots & a_{k+1} & \ldots
\end{array}\right)
$$

that is part of the mapping $u$.

If there are any further elements of $X$ which are not in the domain of $\lambda$, then we select one of them and proceed as before. We obtain another link $\kappa$, say, which is either a finite or a forward link, and, because $u$ is a bijection no element in its domain or range can be an element of the domain or range, respectively, of $\lambda$. Moreover, any element of the range of $\lambda$ is either in the domain of $\lambda$ or belongs to $Z$ (and so belongs to the domain of no part of $u$ ). So the range of $\lambda$ does not meet the domain of $\kappa$, and vice versa. Hence $\lambda$ and $\kappa$ are strongly disjoint. We continue like this until $X$ is exhausted.

If all the elements of $Z$ have not been used up, we select one of them and then proceed as above, but for the mapping $u^{-1}$ instead of $u$. In other words, we take the inverses of the links we have already isolated from $u$, giving finite links and backward links of $u^{-1}$, and then, starting from unused elements of $Z$, we obtain by the above process any further finite links and forward links of $u^{-1}$. There can be no finite links left, because their inverses are finite links of $u$, and we have obtained all of these. The forward links we obtain determine the backward links of $u$.

If any elements of $A$ remain unused, they must be elements of $Y$; and their images under $u$ must also be elements of $Y$; and none of these elements left unused has occurred either in the domain or the range of the links, forward, backward or finite, that we have so far constructed. So the set of elements of $Y$ yet to be used must be mapped bijectively upon itself by $u$. Thus this remaining action of $u$ is a permutation and is strongly disjoint from each link.

LEMMA 2. Let $\lambda_{i}, i=1,2, \ldots, k$, be finite links, of lengths $r_{1} \leqslant r_{2} \leqslant \cdots \leqslant r_{k}$, respectively, that are pairwise strongly disjoint. Let $u=\lambda_{1} \cup \lambda_{2} \cup \cdots \cup \lambda_{k}$ and set $\lambda_{k}=\mu$. Then the inverse semigroups $[u]$ and $[\mu]$ are isomorphic.

Proof. Set $\lambda_{1} \cup \lambda_{2} \cup \cdots \cup \lambda_{k-1}=\sigma$. Thus $u=\sigma \cup \mu=\mu \cup \sigma$. Set $u^{-1}=v$, $\sigma^{-1}=\tau, \mu^{-1}=\nu, r_{k}=r$. Let $f:[\mu] \rightarrow[u]$ be the mapping

$$
\nu^{k} \mu^{\prime} \nu^{m} \mapsto v^{k} u^{l} v^{m}
$$

where $(k, l, m)$ satisfies (1.2). We first show that this is a well-defined mapping. 
Suppose that

$$
\mu=\left(\begin{array}{llll}
a_{1} & a_{2} & \cdots & a_{r-1} \\
a_{2} & a_{3} & \cdots & a_{r}
\end{array}\right)
$$

so that

$$
\nu=\left(\begin{array}{ccc}
a_{r} & \cdots & a_{2} \\
a_{r-1} & \cdots & a_{1}
\end{array}\right)
$$

Then

$$
\begin{aligned}
\nu^{k} \mu^{l} \nu^{m} & =\left(\begin{array}{ccc}
a_{r} & \cdots & a_{k+1} \\
a_{r-k} & \cdots & a_{1}
\end{array}\right)\left(\begin{array}{ccc}
a_{1} & \cdots & a_{r-l} \\
a_{1+l} & \cdots & a_{r}
\end{array}\right)\left(\begin{array}{ccc}
a_{r} & \cdots & a_{m+1} \\
a_{r-m} & \cdots & a_{1}
\end{array}\right) \\
& =\left(\begin{array}{ccc}
a_{r-(l-k)} & \cdots & a_{k+1} \\
a_{r-l} & \cdots & a_{1}
\end{array}\right)\left(\begin{array}{ccc}
a_{1} & \cdots & a_{r-1} \\
a_{1+l} & \cdots & a_{r}
\end{array}\right)\left(\begin{array}{ccc}
a_{r} & \cdots & a_{m+1} \\
a_{r-m} & \cdots & a_{1}
\end{array}\right) \\
& =\left(\begin{array}{ccc}
a_{r-(l-k)} & \cdots & a_{k+1} \\
a_{r-l} & \cdots & a_{1}
\end{array}\right)\left(\begin{array}{ccc}
a_{1} & \cdots & a_{r-l} \\
a_{1+l} & \cdots & a_{r}
\end{array}\right)\left(\begin{array}{ccc}
a_{r} & \cdots & a_{l+1} \\
a_{r-m} & \cdots & a_{l+1-m}
\end{array}\right) \\
& =\left(\begin{array}{ccc}
a_{r-(l-k)} & \cdots & a_{k+1} \\
a_{r-m} & \cdots & a_{1+(l-m)}
\end{array}\right),
\end{aligned}
$$

and this holds for $l \leqslant r-1$. If $l \geqslant r$, then $\nu^{k} \mu^{l} \nu^{m}=\square$, the empty mapping.

Now it follows from this equation that, if $\nu^{k} \mu^{l} \nu^{m}=\nu^{k^{\prime}} \mu^{l^{\prime}} \nu^{m^{\prime}}$, where $k, l, m$ and $k^{\prime}, l^{\prime}, m^{\prime}$ both satisfy (1.2), then this equation holds independently of the value of $r$, provided that $r>l$. If $r \leqslant l$, the equation necessarily holds, since then both sides equal $\square$.

Thus, since $r=r_{k} \geqslant r_{k-1} \geqslant \cdots \geqslant r_{1}$, the equality $\nu^{k} \mu^{l} \nu^{m}=\nu^{k^{\prime}} \mu^{l^{\prime}} \nu^{m^{\prime}}$ implies that $\tau^{k} \sigma^{\prime} \tau^{m}=\tau^{k^{\prime}}{ }^{l^{\prime}} \tau^{m^{\prime}}$. Hence the mapping $f$ is well-defined.

Clearly $f$ is a morphism by Lemma 2.3 , and by Theorem 1.1 it is onto $[u]$. Suppose that $\left(\nu^{k} \mu^{l} \nu^{m}\right) f=\left(\nu^{k^{\prime}} \mu^{l^{\prime}} \nu^{m^{\prime}}\right) f$. Then, since $\sigma$ is strongly disjoint from $\mu$, we must have $\nu^{k} \mu^{\prime} \nu^{m}=\nu^{k^{\prime}} \mu^{l^{\prime}} \nu^{m^{\prime}}$.

LEMMA 3. Let $u$ be the union of the strongly disjoint forward links $\lambda(i), i \in I$. Let $\lambda$ be any (specific) one of these links. Then $[u]$ is isomorphic to $[\lambda]$.

Proof. Let

$$
\lambda=\left(\begin{array}{cccc}
a_{1} a_{2} & \cdots & a_{n} & \cdots \\
a_{2} a_{3} & \cdots & a_{n+1} & \cdots
\end{array}\right)
$$


and set $\mu=\lambda^{-1}$. Then, taking $k, l, m$ to satisfy (1.2), we have $\mu^{k} \lambda^{\prime} \mu^{m}$

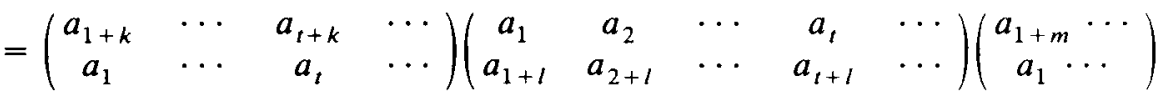

$$
\begin{aligned}
& =\left(\begin{array}{ccc}
a_{1+k} & \cdots & a_{t+k} \\
a_{1+l} & \cdots & a_{t+l}
\end{array} \cdots\right)\left(\begin{array}{cc}
a_{1+m} & \cdots \\
a_{1} & \cdots
\end{array}\right) \\
& =\left(\begin{array}{c}
a_{1+k} \\
a_{1+l}
\end{array} \cdots\right)\left(\begin{array}{cc}
a_{1+l} & \cdots \\
a_{1+l-m} & \cdots
\end{array}\right) \quad(\text { since } m \leqslant l)
\end{aligned}
$$

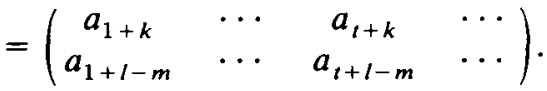

Hence $\mu^{k} \lambda^{\prime} \mu^{m}=\mu^{k^{\prime}} \lambda^{\prime} \mu^{m^{\prime}}$ if and only if $k=k^{\prime}$ and $l-m=l^{\prime}-m^{\prime}$. This applies for each of the forward links $\lambda(i)$. Hence

$$
\mu^{k} \lambda^{l} \mu^{m} \mapsto v^{k} u^{l} v^{m}
$$

where $v=u^{-1}$, is an isomorphism.

Part of what has been proved above is worth isolating for future use.

Lemma 4. Let $\lambda$ be a forward link and set $\mu=\lambda^{-1}$. Then, if $k, l, m$ and $k^{\prime}, l^{\prime}, m^{\prime}$ each satisfy conditions (1.2), we have

if and only if $k=k^{\prime}$ and $l-m=l^{\prime}-m^{\prime}$.

$$
\mu^{k} \lambda^{l} \mu^{m}=\mu^{k^{\prime}} \lambda^{\prime} \mu^{m^{\prime}}
$$

As a dual to Lemma 3, we have

LEMMA 5. Let $u$ be the union of the strongly disjoint backward links $\mu(i), i \in I$. Let $\mu$ be any specific one of these links. Then $[u]$ is isomorphic to $[\mu]$.

Again we wish to note the analogue to Lemma 4.

LEMMA 6. Let $\mu$ be a backward link and set $\lambda=\mu^{-1}$. Then, if $k, l, m$ and $k^{\prime}, l^{\prime}, m^{\prime}$ each satisfy conditions (1.2), we have

$$
\lambda^{k} \mu^{l} \lambda^{m}=\lambda^{k^{\prime}} \mu^{l^{\prime}} \lambda^{m^{\prime}}
$$

if and only if $m=m^{\prime}$ and $l-k=l^{\prime}-k^{\prime}$.

THEOREM 1. Let $u$ be the union of strongly disjoint finite links of unbounded lengths. Then $[u]$ is a free inverse semigroup.

ProOF. If

$$
\mu=\left(\begin{array}{ccc}
a_{1} & \cdots & a_{r-2} \\
a_{2} & \cdots & a_{r}
\end{array}\right)
$$


and $\nu=\mu^{-1}$, as in the proof of Lemma 2, then, with $k, l, m$ satisfying (1.2), we have

$$
\nu^{k} \mu^{l} \nu^{m}=\left(\begin{array}{ccc}
a_{r-(l-k)} & \cdots & a_{k+1} \\
a_{r-m} & \cdots & a_{1+(l-m)}
\end{array}\right) .
$$

Hence, if $r$ is large enough, we have $\nu^{k} \mu^{l} \nu^{m}=\nu^{k^{\prime}} \mu^{\prime} \nu^{m^{\prime}}$ if and only if

$$
l-k=l^{\prime}-k^{\prime}, \quad m=m^{\prime}, \quad k=k^{\prime}, \quad l-m=l^{\prime}-m^{\prime},
$$

i.e. if and only if $(k, l, m)=\left(k^{\prime}, l^{\prime}, m^{\prime}\right)$.

Now, setting $v=u^{-1}$, we have $v^{k} u^{l} v^{m}=v^{k^{\prime}} u^{\prime} v^{m^{\prime}}$ if and only if the corresponding equation holds for each of the strongly disjoint links whose union is $u$. Since these are of unbounded length, it follows that $k=k^{\prime}, l=l^{\prime}, m=m^{\prime}$. Hence $[u]$ is isomorphic to $G A$ (or $G B$ or $S C$ ), which we have seen to be free.

A point from the proof of Lemma 2 is perhaps worth emphasising: namely we have obtained Corollary 5 of Djadčenko and Saǐn (1974).

THEOREM 2. Let $\lambda$ be a link of length $r$. Then $[\lambda]$ has $1+2^{2}+3^{2}+\cdots+r^{2}$ $=\frac{1}{6} r(r+1)(2 r+1)$ elements.

Proof. The proof of Lemma 2 involved showing, incidentally, that the elements $v^{k} u^{l} v^{m}$ for $l \leqslant r-1$ were all distinct. For $l=r, \lambda^{r}$ is the empty mapping. The result follows.

A second point worth noting is

THEOREM 3. Let $\lambda$ be a forward link and $\mu$ a backward link. Then $[\lambda] \cong[\mu]$, and each is isomorphic to the bicycle semigroup.

Proof. $[\mu]$ is equally generated by $\mu^{-1}$, and $\mu^{-1}$ is a forward link. Hence $[\mu] \cong[\lambda]$.

Suppose that

$$
\lambda=\left(\begin{array}{cccc}
a_{1} a_{2} & \cdots & a_{k} & \cdots \\
a_{2} a_{3} & \cdots & a_{k+1} & \cdots
\end{array}\right) .
$$

Then $\lambda \lambda^{-1}=1$, the identity on $\left\{a_{1}, a_{2}, \ldots\right\}$, and so an identity for the semigroup $[\lambda]$. Moreover $\lambda^{-1} \lambda=1_{A}$, the identity on $A$, where $A=\left\{a_{2}, a_{3}, \ldots\right\}$. So $\lambda^{-1} \lambda<$ $\lambda \lambda^{-1}=1$. Hence, by Lemma 1.31 of Clifford and Preston (1961), $[\lambda]$ is a bicyclic semigroup.

We can also use the techniques developed to prove

THEOREM 4. Let $\lambda$ be a forward link and $\sigma$ a backward link strongly disjoint from $\lambda$. Let $u=\lambda \cup \sigma$. Then $[u]$ is a free inverse semigroup. 
Proof. Set $v=u^{-1}, \mu=\lambda^{-1}, \tau=\sigma^{-1}$. Then $v^{k} u^{l} v^{m}=\mu^{k} \lambda^{\prime} \mu^{m} \cup \tau^{k} \sigma^{l} \tau^{m}$. The elements of $[u]$ can all be written in the form $v^{k} u^{l} v^{m}$ with $k, l, m$ satisfying conditions (1.2). It will suffice to prove that no two such elements are equal.

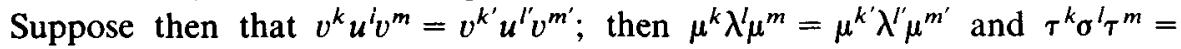
$\tau^{k^{\prime}} \sigma^{l^{\prime}} \tau^{m^{\prime}}$

From Lemma 4, the first of these equations implies that $k=k^{\prime}$ and $l-m=$ $l^{\prime}-m^{\prime}$. Taking inverses in the second equation to give $\sigma^{m} \tau^{\prime} \sigma^{k}=\sigma^{m^{\prime}} \tau^{\prime} \sigma^{k^{\prime}}$, and again applying Lemma 4, we obtain $m=m^{\prime}$ and $l-k=l^{\prime}-k^{\prime}$. Alternatively, we may use Lemma 6 . Hence we have $(k, l, m)=\left(k^{\prime}, l^{\prime}, m^{\prime}\right)$.

THEOREM 5. Let $\lambda$ be a forward link and let $\mathrm{o}$ be a permutation strongly disjoint from $\lambda$. Then $[\lambda \cup \sigma] \cong[\lambda]$. The result also holds if $\lambda$ is a backward link.

Proof. Set $u=\lambda \cup \sigma, v=u^{-1}, \mu=\lambda^{-1}$. As in Theorem 4, it suffices to show that, for $k, l, m$ and $k^{\prime}, l^{\prime}, m^{\prime}$ each satisfying conditions (1.2), $v^{k} u^{\prime} v^{m}=v^{k^{\prime}} u^{\prime} v^{m^{\prime}}$ if and only if $\mu^{k} \lambda^{\prime} \mu^{m}=\mu^{k^{\prime}} \lambda^{\prime} \mu^{m^{\prime}}$.

Suppose $\mu^{k} \lambda^{\prime} \mu^{m}=\mu^{k^{\prime}} \lambda^{l^{\prime}} \mu^{m^{\prime}}$. Then, by Lemma $4, k=k^{\prime}$ and $l-m=l^{\prime}-m^{\prime}$. Hence

$$
\begin{aligned}
v^{k} u^{l} v^{m} & =\mu^{k} \lambda^{\prime} \mu^{m} \cup \sigma^{-k} \sigma^{\prime} \sigma^{-m} \\
& =\mu^{k} \lambda^{\prime} \mu^{m} \cup \sigma^{l-k-m} \\
& =\mu^{k^{\prime}} \lambda^{\prime} \mu^{m^{\prime}} \cup \sigma^{l^{\prime-k^{\prime}-m^{\prime}}} \\
& =v^{k^{\prime}} u^{\prime} v^{m^{\prime}} .
\end{aligned}
$$

The reverse implication holds because $\lambda$ and $\sigma$ are strongly disjoint.

As an extension of the isomorphism given in Theorem 3, we have

THEOREM 6. Let $\lambda$ be a forward link, $\mu$ a backward link, $\phi$ a finite link of length $r$ strongly disjoint from $\lambda$ and $\psi$ a finite link of length $r$ strongly disjoint from $\mu$. Then $[\phi \cup \lambda] \cong[\psi \cup \mu]$.

Proof. As for Theorem 3 this result follows immediately from the observation that $[\phi \cup \lambda]$ is also generated by $(\phi \cup \lambda)^{-1}$.

The result of the next lemma we have to use several times.

LEMMA 7. Let $u, v, w$ be strongly disjoint bijections such that $[u \cup v] \cong[v]$. Then $[u \cup v \cup w] \cong[v \cup w]$.

Proof. Since $w$ is strongly disjoint from $u \cup v$, the isomorphism between $[u \cup v]$ and $[v]$ extends immediately to an isomorphism between $[u \cup v \cup w]$ and 
$[v \cup w])$, if we use the fact that $(u \cup v \cup w)^{-k}(u \cup v \cup w)^{l}(u \cup v \cup w)^{-m}=$ $(u \cup v)^{-k}(u \cup v)^{l}(u \cup v)^{-m} \cup w^{-k} w^{l} w^{-m}$, and if we note that this latter union is also strongly disjoint.

\section{As an immediate corollary we have}

LeMMA 8. Let $u$ be a bijection that generates a free inverse semigroup. Let $w$ be a bijection strongly disjoint from $u$. Then $[u \cup w]$ is free.

We have now in fact isolated all the distinct isomorphism types of monogenic inverse semigroups. We state this as the next theorem, but first define the types. In these definitions $r$ denotes an integer $\geqslant 1$ giving the length of a finite link involved in the definition, and $s$ denotes an integer $\geqslant 1$ or can be infinite, denoted $\infty$, being the order of an element of a group.

Type $(r, s)$ : isomorphic to an inverse semigroup generated by the strongly disjoint union of a finite link of length $r$ and a permutation of order $s$. If $u$ is such a union, then the semigroup $\langle u\rangle$ (in constrast to the inverse semigroup $[u]$ ) is a cyclic (i.e. monogenic) semigroup of index $r$ and period $s$ (in the terminology of Clifford and Preston (1961)). So we call $r$ the index and $s$ the period of a semigroup of type $(r, s)$.

Type ( $r$, Fwd): isomorphic to an inverse semigroup generated by a finite link of length $r$ and a strongly disjoint forward link (or, by Theorem 6, a finite link of length $r$ and a strongly disjoint backward link).

Type FI: a free monogenic inverse semigroup.

THEOREM 7. Let $[u]$ be a monogenic inverse semigroup, generated by $u$. Then $[u]$ is of one of the types $(r, s),(r, F w d), F I$. Moreover, these isomorphism types are distinct.

Proof. By taking some faithful representation of $[u]$ we can suppose that $u$ is a bijection. Hence, by Lemma 1, $u$ can be decomposed, uniquely, into a strongly disjoint union of a set $\left\{\phi_{i} \mid i \in I\right\}$ of finite links, a set $\left\{\lambda_{j} \mid j \in J\right\}$ of forward links, a set $\left\{\mu_{k} \mid k \in K\right\}$ of backward links and a permutation $\sigma$, say. Set $\cup \phi_{i}=\phi$, $\cup \lambda_{j}=\lambda$ and $\bigcup \mu_{k}=\mu$.

Because of Lemmas 3 and 7 , the isomorphism type of $[u]$ is unchanged if, when $J \neq \square$, we replace $\lambda$ by any one of the $\lambda_{j}$. We do this and may assume therefore that either $J$ is empty or that $J=\{1\}$. Similarly, by Lemmas 5 and 7, we may assume that either $K$ is empty or $K=\{1\}$.

For the $\phi_{i}$ there are two possibilities: either there is a $\phi_{i}$ of maximal length or not. In the first event, by Lemmas 2 and 7, we may, without changing the isomorphism type of $[u]$, replace the set of $\phi_{i}$ by any single $\phi_{i}$ which has maximal 
length. In the second event, by Lemma 8, we may, again preserving the isomorphism type of $[u]$, replace the $\phi_{i}$ by a subsequence of finite links of unbounded lengths.

Let us deal with this final case first: by Lemma 8 and Theorem $1,[u]$ is then isomorphic to $[\phi]$, a union of a sequence of finite links of unbounded lengths, and is free, i.e. of type $F I$.

There is another case leading to this isomorphism type, namely that when $J$ and $K$ are each non-empty. In that event, as we have assumed, $u$ contains the bijection $\lambda_{1} \cup \mu_{1}$ which, by Theorem 4, generates a free inverse semigroup. Hence, by Lemma $8,[u]$ is of type $F I$.

For the remaining cases, consider first when $J$ and $K$ are both empty; then $[u] \cong[\phi \cup \sigma]$, where $\phi$ is a single finite link. So $[u]$ is of type $(r, s)$, where $r$ is the length of $\phi$ and $s$ is the order of $\sigma$. We can always assume that $\phi$ and $\sigma$ are both present, if necessary, by taking $r=1$, so that $\phi$ is the empty mapping, or by taking $\sigma$ to be the permutation of the empty set.

Suppose then that $J=\{1\}$ and $K$ is empty. Thus $u=\phi \cup \lambda_{1} \cup \sigma$, a strongly disjoint union of a finite link, a forward link and a permutation. By Theorem 5 , $\left[\lambda_{1} \cup \sigma\right] \cong\left[\lambda_{1}\right]$. Hence by Lemma $7,[u] \cong\left[\phi \cup \lambda_{1}\right]$ and so is of type $(r$, Fwd $)$, where $r$ is the length of $\phi$.

If $K=\{1\}$ and $J$ is empty, then similarly $[u] \cong[\phi \cup \mu]$, where $\phi$ is a finite link of length $r$, say, and where $\mu$ is a strongly disjoint backward link. By Theorem 6 , $[u]$ is therefore of type ( $r$, Fwd).

We have dealt with all possibilities.

It remains to show that the isomorphism types are distinct. We do this by appealing to equations that hold in the various types that Gluskin (1957) and Eršova (1961) have in fact used to characterize the types.

Let $u=\phi \cup \lambda$, a strongly disjoint union of a finite link $\phi$ of length $r$ and a forward link $\lambda$. Set $v=u^{-1}$. Then it is easily calculated that

$$
u^{r} u v=u^{r}
$$

but that

$$
u^{s} u v \neq u^{s}, \quad \text { if } 1 \leqslant s<r .
$$

Equation (1) also immediately implies, by left multiplying by the apprpriate power of $u$, that

$$
u^{t} u v=u^{t}, \quad \text { if } t \geqslant r .
$$

Equation (3), combined with the inequalities (2), immediately leads to the conclusion that the isomorphism types $(r$, Fwd $)$ are distinct for distinct values of $r$.

Again our representation of a free monogenic inverse semigroup as, for example, a strongly disjoint union of a sequence of finite links of unbounded 
lengths immediately shows that no equation (1) is satisfied in a semigroup of type FI.

Consider now those of type $(r, s)$ for $s$ finite, so that we may take $u=\phi \cup \sigma$ as a generator, where $\phi$ is a finite link of length $r$ and $\sigma$ is a permutation of finite order $s$. Then it is easily calculated that

$$
u^{r+s}=u^{r}
$$

and that $r+s$ is the least power of $u$ equal to a smaller power, so that both $r$ and $s$ are uniquely determined by this equation. It follows immediately, for $s$ finite, that semigroups of types $(r, s)$ and $\left(r^{\prime}, s^{\prime}\right)$ are isomorphic if and only if $r=r^{\prime}$ and $s=s^{\prime}$. Moreover, it is easily calculated that no equation (4) is satisfied in a semigroup of type ( $r, \mathrm{Fwd}$ ) or of type $\boldsymbol{F I}$.

It remains to consider those of type $(r, \infty)$. Suppose $u=\phi \cup \sigma$, where $\phi$ is a finite link of length $r$ strongly disjoint from $\sigma$, a permutation of infinite order. Set $v=u^{-1}$, as before. Then, by an easy calculation, we have

$$
v u u^{r}=u^{r} u v
$$

and

$$
v u u^{s} \neq u^{s} u v, \quad 1 \leqslant s<r .
$$

Hence, immediately, monogenic inverse semigroups of type $(r, \infty)$, for distinct $r$, are not isomorphic.

Our representations of semigroups of type $F I$ immediately show that no equations (5) hold in these semigroups.

We know that in an inverse semigroup of type ( $r$, Fwd) equation (1) holds; but it is quickly checked that $v u u^{s}=u^{s}$ holds for no $s \geqslant 1$ when $u$ is the strongly disjoint union of a finite link and a forward link. Hence, in particular, equation (5) does not hold.

The proof of the theorem is complete.

COROLLARY (TO THE PROOF). The distinct types of monogenic inverse semigroup [u] can be characterized as follows (where $v=u^{-1}$ ):

Type $(r, s), s$ finite: satisfy $u^{r+s}=u^{r}$, with $r+s$ the minimum such natural number.

Type $(r, \infty)$ : satisfy vuu ${ }^{r}=u^{r} u v$, with $r$ the least such natural number.

Type ( $r$, Fwd): satisfy either $(a) u^{r} u v=u^{r}$, with $r$ the least such natural number, and with $v u u^{s} \neq u^{s}$ for all $s \geqslant 1$; or $(b) v u u^{r}=u^{r}$, with $r$ the least such natural number, and with $u^{s} u v \neq u^{s}$ for all $s \geqslant 1$.

Type FI: satisfy for no r or $s \geqslant 1$, any of the equations satisfied by the other types.

Proof. The only point perhaps requiring comment is that alternative characterizations are given to type $(r$, Fwd). This results from Theorem 6 ; for a semigroup of type $(r$, Fwd $)$ is generated by an element that can be regarded as a 
strongly disjoint union of a finite link and either a forward link or a backward link. Characterization (a) results from taking the first alternative, and (b) from the second alternative.

The proof of the corollary is complete.

Of course each of (a) and (b) in the above corollary results from the other by replacing $u$ by its inverse. In this sense the other characterizations, for types $(r, s)$, are self-dual.

It is also worth noting that the representations that we have obtained for the different types enable us quickly to calculate that $x^{r+s}=x^{r}$ is an identity for monogenic inverse semigroups of type $(r, s)$, with $s$ finite, and that $x^{-1} x x^{r}=$ $x^{r} x x^{-1}$ is an identity for those of type $(r, \infty)$. (This latter is also an identity for type $(1, s)$, with $s$ finite.) Hence, the monogenic inverse semigroups of type $(r, s)$, with $s$ finite or infinite, are free inverse semigroups with one generator in the variety of inverse semigroups determined, respectively, by these identities.

The equation $x^{r} x x^{-1}=x^{r}$ is not an identity for monogenic semigroups of type ( $r$, Fwd); for if it were, then $v^{r} v u=v^{r}$ would hold, which by taking inverses gives $u^{r}=v u u^{r}$, a contradiction. A similar statement holds for the alternative characterization (b) of the corollary.

\section{Addendum}

Since this paper was written, J. B. Conway, J. Duncan and A. L. T. Paterson, in Section 1 of their paper (1984), 'Monogenic inverse semigroups and their $C^{*}$. algebras', have obtained the same classification results for monogenic inverse semigroups, by using results from operator theory on the decomposition of partial power isometries on a Hilbert space.

\section{References}

A. H. Clifford and G. B. Preston (1961), The algebraic theory of semigroups, Volume 1 (Mathematical Surveys No. 7, Amer. Math. Soc., Providence, R. I.).

J. B. Conway, J. Duncan and A. L. T. Paterson (1984), 'Monogenic inverse semigroups and their $C^{*}$-algebras', Proc. Roy. Soc. Edinburgh Sect. A 98, 13-24.

G. G. Djadčenko and B. M. Šăn (1974), 'The structure of finite monogenic inverse semigroups', Ural. Gos. Univ. Mat. Zap. 9, 15-21.

M. L. Dubreil-Jacotin, L. Lesieur and R. Croisot (1953), Théorie des treillis, des stuctures algébriques ordonnees, et des treillis géométriques (Gauthier-Villars, Paris).

Carl Eberhart and John Seldon (1972), 'One-parameter inverse semigroups', Trans. Amer. Math. Soc. 168, 53-66.

T. I. Eršova (1971), 'Monogenic inverse semigroups', Ural. Gos. Univ. Mat. Zap. 8, 30-33.

L. M. Gluskin (1957), 'Elementary generalized groups', Mat. Sb. 41 (83), 23-36. 
L. M. Gluskin (1961), 'On inverse semigroups', Zap. Meh.-Mat. Fakulty, i Har'kov Mat. Ob̌̌č. (4) 28, 103-110.

D. B. McAlister (1973), Review of Eberhart and Selden, 'One-parameter inverse semigroups', Math. Rev. 45, \#5258.

D. B. McAlister (1974), 'Groups, semilattices and inverse semigroups', Trans. Amer. Math. Soc. 192, 227-244.

D. B. McAlister and R. McFadden (1974), 'Zig-zag representations of inverse semigroups', J. Algebra 32, 178-206.

W. D. Munn (1957), 'Characters of the symmetric inverse semigroup', Proc. Cambridge Phil. Soc. 53, 13-18,

W. D. Munn (1974), 'Free inverse semigroups', Proc. London Math. Soc. 29, 385-404.

G. B. Preston (1973), 'Free inverse semigroups', J. A ustral. Math. Soc. 16, 443-453.

H. E. Scheiblich (1973), 'Free inverse semigroups', Proc. Amer. Math. Soc. 38, 1-7.

Department of Mathematics

Monash University

Clayton, Victoria 3168

Australia 